\title{
Helicobacter pylori Pathogenicity Islands and Giardia lamblia Cysteine Proteases in Role of Coinfection and Pathogenesis
}

\author{
Mihret Tilahun (1D) \\ Alemu Gedefie (iD) \\ Chernet Belayhun ${ }^{2}$ \\ Zenawork Sahle (1D ${ }^{3}$ \\ Admasu Abera ${ }^{3}$ \\ 'Department of Medical Laboratory \\ Sciences, College of Medicine and Health \\ Science, Wollo University, Dessie, \\ Ethiopia; ${ }^{2}$ Department of Medical \\ Laboratory Science, Mehal Meda \\ Hospital, North Showa, Ethiopia; \\ ${ }^{3}$ Department of Medical Laboratory \\ Science, Debre Birhan Health Science \\ College, North Showa, Ethiopia
}

Correspondence: Mihret Tilahun Department of Medical Laboratory Science, College of Medicine and Health Sciences, Wollo University, PO.BOX:

I 145, Dessie, Ethiopia

Tel +25I 920988307

Fax +25I 333II5250

Email tilahunmihret2I@gmail.com

\begin{abstract}
Helicobacter pylori is a well-known human-specific stomach pathogen that infects more than half of the world's population. The infection with this bacterium can cause a variety of gastrointestinal problems, including chronic gastritis, peptic ulcers, and even cancer. H. pylori is a highly infectious bacterium. H. pylori causes an increase in gastric mucosa $\mathrm{pH}$ or gastric mucosa intestinal metaplasia. These modifications in the stomach environment are necessary for $G$. lamblia colonization to occur. Giardia lamblia is a flagellate protozoan parasite that can cause giardiasis in humans and other mammals. It dwells in the duodenum and upper jejunum. Globally, over 280 million cases of human giardiasis are predicted to occur each year. Simultaneous human colonization by G. lamblia and $H$. pylori is a typical occurrence since the viruses' predisposing factors are similar in both groups. Giardiasis is a parasitic infection that affects both children and adults worldwide. Infection with Giardia is more common in underdeveloped countries. Globally, more than 200 million cases of giardiasis are detected each year. In contrast, the presence of G. lamblia in the host body triggers an immunological response comparable to that of H. pylori, with lymphocytes strongly polarized towards Th1. As a result, their combined presence exacerbates host tissue damage. The major goal of this seminar is to describe the pathophysiology, immunology, and clinical aspects of G. lamblia and H. pylori coinfection using a comprehensive search of PubMed, Lancet, and Google Scholar sources. Upper gastrointestinal problems such as upper abdominal pain, abdominal bloating, nausea, vomiting, epigastric pain/burning, and belching are all caused by both organisms. Differentiation by physical examination is impossible in people infected with both bacteria. For this coinfection distinction, a laboratory diagnosis is required. G. lamblia and H. pylori, when present together, have a synergistic effect on the host and can cause serious damage. As a result, researchers should delve deeper into the mechanics underlying this potential microbial interaction.
\end{abstract}

Keywords: Helicobacter pylori, pathogenicity islands, Giardia lamblia, coinfection

\section{Introduction}

$H$. pylori is a spiral-shaped gram-negative human pathogen that colonizes the stomach's antrum and corpus. In the last decade, numerous virulence factors have been identified. These elements allow the bacterium to live in the gastrointestinal tract's highly acidic environment, reach the more neutral environment of the mucous layer, and withstand the human immune response, resulting in persistence. Majority of the infections happen in the childhood age, with just a small percentage of infections progressing to severe conditions. ${ }^{1}$ Helicobacter pylori is a well-known 
human-specific gastrointestinal pathogen that infects more than half of the world's population. Infection with this bacterium can cause a variety of gastrointestinal problems, including chronic gastritis, peptic ulcers, and even cancer. H. pylori is a highly infectious bacterium. The cag (cytotoxin-associated genes) pathogenicity island, a 40-kb stretch of DNA that encodes components of the type IV secretion system, is found in H. pylori isolates (T4SS). This T4SS forms a pilus for the injection of virulence factors into host target cells such as the CagA oncoprotein. ${ }^{2}$ The function of the Helicobacter pylori cagA protein, the only $H$. pylori protein that has been identified as being transported into host gastric epithelial cells via a type IV secretion system, in the development of H. pylori-associated illnesses, such as gastric cancer. ${ }^{3}$ Gastric carcinoma is the world second leading cause of cancer-related deaths, accounting for over 400,000 deaths each year. Infection with cagA-positive Helicobacter pylori has an important role in the development of stomach cancer. The cagA gene product CagA is delivered directly into gastric epithelial cells by the type IV secretion system. ${ }^{4}$

Giardia duodenalis (syn. G. intestinalis, G. lamblia), the etiological agent of Giardiasis, is one of the most common intestinal protozoan flagellates in humans. Giardia species have a simple life cycle that consists of two active trophozoites and cystic forms. This parasite spreads through direct or indirect ingestion of infectious cysts via the fecal-oral route. After ingesting cysts, the incubation period ranges from 9 to 15 days. ${ }^{5-7}$ Giardiasis is a parasitic infection that affects both children and adults worldwide. Infection with Giardia is more common in underdeveloped countries. Globally, more than 200 million cases of giardiasis are detected each year. Since 2004, Giardia has been part of the World Health Organization's neglected diseases initiative. ${ }^{8}$ Around about 200 million of people in the world are with clinically manifested giardiasis, With a global illness burden of around 280 million cases each year. ${ }^{9,10}$ A countrywide survey of giardiasis in Ethiopia, using formal ether concentration method, among school children (5-19 years of age) and residents showed overall prevalence rates of $8.9 \%$ and $3.1 \%$ respectively. ${ }^{11}$

The acidic environment in the stomach and the presence of bile and trypsin in the duodenum stimulate infectious cysts to excyst, and infectious cysts in contaminated food or water, or directly through the fecal-oral route, are transferred through ingestion of infectious cysts in contaminated food or drink. Then they change into trophozoites, which use the sticky disc to connect to intestinal epithelial cell. ${ }^{12,13}$ Coinfections are common in places with inadequate sanitation and/or incorrect drinking water treatment, as the oral route of Giardia infection is shared with many other gastrointestinal (GI) pathogens. G. lamblia coinfections with pro-inflammatory pathogens such as Helicobacter pylori, Vibrio cholerae, and Escherichia coli are widespread and reported concurrently because this route of infection is shared by a wide range of gastrointestinal pathogens. ${ }^{5,14,15}$

G. intestinalis and $H$. pylori are known to infect humans' gastrointestinal tracts early life and to be quite common in endemic locations throughout their lives. Giardia causes diarrhea, bloating, flatulence, and malnutrition, whereas H. pylori colonizes the gastric mucosa and can cause peptic ulcers, chronic gastritis, and gastric cancer. G. intestinalis has a lot of genetic diversity, with two genotypes or assemblages (A and B) infecting people. Differences in symptoms have also been linked to these two separate genetic types of individuals. ${ }^{16,17}$

In an Indian study, the presence of G. lamblia trophozoites was confirmed in biopsies from 54 stomach cancer patients and 100 peptic ulcer patients. Protozoa were found in $14.9 \%(8 / 54)$ and $20 \%(7 / 35)$ of the cases, respectively. There was a strong link between the presence of G. lamblia in stomach samples and the presence of H. pylori, with $6 / 7$ people with peptic ulcers and all cases of gastric cancer coexisting with these microbes. ${ }^{18}$

People infected with G. lamblia had a threefold higher likelihood of co-occurrence with $H$. pylori in children from Sub-Saharan Africa, according to research (12.2\% vs $30.2 \%$ ). Furthermore, children aged one to five years old had the highest frequency of $G$. lamblia isolation $(28.7 \% ; 43 / 150) .{ }^{19}$ The prevalence of those coinfections varies by region in Ethiopia. For example, a study conducted at Mekane Selam Hospital in South Wollo found that $H$. pylori infected participants had a higher proportion of intestinal parasites (44.3\%). G. lamblia was responsible for $26 \%$ of the coinfected parasites. ${ }^{20}$

Predisposing factors are coincident in both groups of these pathogens, such as low age, immune suppression status, low socioeconomic and educational status, and consumption of contaminated water sources, and they are upper gastrointestinal complaints such as upper abdominal pain, abdominal bloating, nausea, vomiting, and epigastric bleeding. ${ }^{21,22}$ In patients coinfected with those pathogens differentiation by physical examination is not possible. So, 
laboratory diagnosis is recommended. A sanitary life style as well as safe water source would prevent most of those infections worldwide. ${ }^{23}$ The aim of this review was to describe the pathogenesis, immunology and clinical features of Giardia lamblia and Helicobacter pylori coinfection from different literatures.

\section{Methodology}

Literatures written on pathogenesis, immunology and clinical features of Helicobacter pylori and Giardia lamblia coinfection from the year 1995-2021 were systematically searched and reviewed using Pub med, lancet, PMC and Google scholar sources.

\section{Helicobacter pylori Pathogenicity Islands and Giardia lamblia Cysteine Proteases in Role of Coinfection and Pathogenesis \\ Pathogenesis}

The two flagellate microorganisms, Giardia lamblia and Helicobacter pylori, colonize the gastrointestinal tract of their human hosts in close proximity. Giardia trophozoites can colonize and replicate in rapidly in the human gastrointestinal tract, causing symptoms in the process. Giardia can also infect the gall bladder, where bile serves as a major growth factor stimulant. It is thought to have a strong predilection for a $\mathrm{pH}$ range of $6.38-7.02 .^{24}$ In the general population, gastrointestinal complaints are very common. Dyspepsia is the most prevalent upper gastrointestinal symptom complex, with dyspeptic symptoms affecting $25 \%$ of the general population. The causes of dyspepsia are unknown, however infectious and noninfectious agents are likely to be involved. Dyspepsia is caused by Helicobacter pylori (H. pylori), Giardia lamblia (G. lamblia), and celiac disease. ${ }^{25}$ H. pylori is thought to infect more than half of the world's population, ${ }^{20}$ with the highest burden in underdeveloped nations like Africa. $G$. lamblia has a global spread, infecting around 200 million individuals and causing 500,000 new cases each year. ${ }^{26,27}$ In patients with dyspepsia, $30 \%$ of $H$. pylori and $15.8-44 \%$ G. lamblia infection, respectively, were reported. ${ }^{25,28} H$. pylori infection, giardiasis, and amoebiasis were found in $65 \%, 10-25 \%$ of children with stomach symptoms, respectively. ${ }^{29}$ A possible link between intestinal protozoans and $H$. pylori infection has been established in several parts of the world, with $75 \%$ prevalence of $H$. pylori infection among patients with gastric giardiasis. $^{30,31}$

\section{Helicobacter pylori}

At least half of the world population has $H$. pylori in their stomachs. It is most commonly acquired during childhood, and if left untreated, it will last the rest of the host's life. ${ }^{32}$ Helicobacter pylori, an opportunistic stomach pathogen, secretes urease in its immediate environs to raise the $\mathrm{pH}$ of the mucus and so diminish its viscosity, as well as stimulating monocyte and neutrophil chemotaxis. ${ }^{33}$ Gastritis and peptic ulcers are caused by Helicobacter pylori. H. pylori infection is connected to mucosal-associated lymphoid tissue (MALT) lymphomas and is a risk factor for gastric cancer. $^{34} H$. pylori infection has also been linked to the occurrence of stomach MALT lymphomas in epidemiologic research. In addition, elimination of the virus causes most localized gastric MALT lymphomas to disappear. ${ }^{35}$ Although $H$. pylori causes histologic gastritis in all infected people, only a small percentage of them show any clinical indications of colonization. H. pylori-positive patients are said to have a $10 \%$ to $20 \%$ lifetime chance of getting ulcers and a $1 \%$ to $2 \%$ lifetime risk of developing distal gastric cancer. ${ }^{36}$ Gastric cancer is one of the most common cancers in both men and women, and it is the fourth leading cause of cancer-related deaths globally. The interplay of genetic and environmental variables is linked to the etiology of gastric cancer, with Helicobacter pylori (H. pylori) infection being a prominent contributor. Due to multiple evasive mechanisms generated by the bacterium's virulence factors, invasion, survival, colonization, and encouragement of additional inflammation inside the gastric mucosa are feasible. ${ }^{37}$ Helicobacter pylori infects the gastric epithelial cells of at least half of the world's population, and it's the leading cause of gastrointestinal problems such as chronic gastritis, ulcer illnesses, and gastric cancer. The bacteria must survive extreme stomach conditions in order to colonize and create a persistent infection. $H$. pylori has a well-developed mechanism for surviving in an acidic environment. Despite bacterial causes, gastric environmental conditions and host genetic elements work together to make gastric pathogenicity possible. ${ }^{38}$ Although $H$. pylori possess flagella for motility, the added change in viscosity facilitates quick and easy transport through the mucus layers. ${ }^{39}$ This bacterium has the ability to produce virulence factors that help to start and accelerate the spread of certain diseases. For example, the oncoprotein CagA (cytotoxin associated gene A) is linked 
to the immortalization of gastric mucosal cells, the breakdown of tight junctions, and the onset of cell-to-cell contact loss. ${ }^{40}$

CagA inhibits the actions of a recognized $H$. pylori toxin, and it has functions that are distinct from those of conventional toxins. ${ }^{41}$ Another $H$. pylori characterized toxin is VacA (vacuolating cytotoxin A), which contributes to cell apoptosis, promotes inflammation and inhibits the proliferation of $\mathrm{T}$ and $\mathrm{B}$ cells. In addition this pathogen produce large amounts of urease, an enzyme associated with the alkalization of the local environment, as well as outer membrane proteins (OMPs) that enable rapid and efficient colonization of the gastric mucosa. ${ }^{42}$

\section{H. pylori Pathogenicity Islands and Colonization}

H. pylori is a remarkable bacterium in that it may colonize the stomach of untreated humans for the rest of their lives. The stomach colonization, inflammation, altered gastric acid production, and tissue death that are hallmarks of $H$. pylori disease are caused by a variety of reasons. Initial colonization is aided by a bacterial acid-inhibitory protein that blocks acid production and neutralizes gastric acids with ammonia produced by bacterial urease activity. $^{43} H$. pylori's ability to induce this range of disorders is influenced by host, bacterial, and environmental variables. Urease, flagella, adhesins, and -glutamyl transpeptidase are examples of bacterial factors. H. pylori can survive for decades thanks to substances like lipopolysaccharide, urease, and vacuolating cytotoxin, which cause an inflammatory response that damages host cells. The cag pathogenicity island contains genes that activate a signal transduction cascade, resulting in the synthesis of interleukin-8. The inflammation is exacerbated by proinflammatory cytokines and a Th-1 cytokine response. ${ }^{44}$

Helicobacter pylori is a genetically diversified species, and the cytotoxin associated gene pathogenicity island is the most important bacterial virulence factor that raises the likelihood of disease versus asymptomatic colonization (cagPAI). Although socially housed rhesus macaques are frequently infected with $H$. pylori, which is comparable to the $H$. pylori that infects humans, nothing is known about the cagPAI. H. pylori strains derived from naturally infected rhesus macaques exhibit a cagPAI that is remarkably similar to that found in human clinical isolates, and it encodes a functional type IV secretion system, just like human isolates. $^{45}$
An important virulence factor of $H$. pylori Localized tissue damage is urease by products (mucinase and phospholipases), vacuolating cytotoxin A (VacA), cytotoxinassociated gene (cagA), the cag phosphoribosyl anthranilate isomerase (PAI) and Release of proteases and reactive oxygen. ${ }^{46}$ In humans, the pathophysiology of Helicobacter pylori infection may be broken down into three steps: (1) invasion, colonization, and adherence to the human stomach mucosa; (2) avoidance, subversion, or exploitation of the human immune system; and (3) multiplication, tissue damage, and transmission to a new susceptible host. $^{47}$ For H. pylori colonization in the stomach to be successful: there are four phases that must occur for $H$. pylori colonization and pathogenesis to occur: (1) survival in an acidic stomach; (2) flagella-mediated motility toward epithelial cells; (3) adhesin-mediated attachment to host receptors; (4) tissue damage caused by toxin release. These are reflected below:

Step 1: Urease and survival under acidic stomach conditions: The pathogen that causes ulcers in the stomach and intestines known as Helicobacter pylori. Helicobacter pylori is the only bacteria known to colonize the stomach's highly acidic environment. H. pylori lives in acidic environments by manufacturing urease, a protein that catalyses the breakdown of urea to create ammonia, raising the $\mathrm{pH}$ of the environment. ${ }^{48} H$. pylori neutralize stomach acid by generating ammonia from urea utilizing bacterial by urease enzyme. The usage of $H$. pylori's adhesion protein BabA to connect to Lewis's antigens, which are naturally produced sugars on healthy gastric epithelium, is another survival mechanism. ${ }^{49} H$. pylori has developed an acid tolerance system that regulates urease activity to promote periplasmic $\mathrm{pH}$ adjustment in the severe acidic environment of the stomach ${ }^{49}$ The H. pylori bacteria reduces the stomach's and duodenum's protective mucous layer, allowing acid to reach the delicate lining beneath. The acid and germs both irritate the lining, resulting in a sore or ulcer. ${ }^{50}$ By altering the viscoelasticity qualities of stomach mucins, urease activity aids flagellar movement through the mucous layer. Gastric mucins produce a gel that effectively confines bacteria at low $\mathrm{pH}$, while ureasecatalysed ammonium ion synthesis elevates the $\mathrm{pH}$ to near neutral, transitioning the mucous gel to a viscoelastic fluid through which H. pylori can swim. ${ }^{51}$ The altered distribution of chemotaxis mutants associated with decreased inflammation, poor $\mathrm{CD} 4+\mathrm{T}$ cell recruitment, and the absence of a $\mathrm{T}$ helper 17 (TH17) response, in addition to favouring clearance (Study demonstrating that chemotaxis 
promotes interactions between the bacteria and epithelium that drive pro-inflammatory $\mathrm{T} \mathrm{H} 17$ responses).

Step 2: Flagella and movement toward epithelium cells: Helicobacter pylori is a flagellated bacterium that colonizes and leads to inflammation in the human gastroduodenal mucosa, causing gastrointestinal illness. Colonization and virulence factors are thought to be involved in its pathogenesis. The fundamental function of the flagella of Helicobacter pylori is to provide motility. ${ }^{51}$ By the action of 4-7 polar sheathed flagella, H. pylori moves through the stomach mucosa epithelial layer to the basal layer, where the $\mathrm{pH}$ value is near to 7.0. Flagellamediated motility is required for $H$. pylori colonization of the gnotobiotic piglet and mouse stomach mucosa. ${ }^{52}$ Flagella can be thought of as a colonization/virulence component in the early stages. Furthermore, mice immunized with a vaccine enriched for $H$. pylori flagella sheath proteins showed much lower colonization than mice immunized with whole-cell lysate. Flagella can be used as a diagnostic and vaccination target due to the high antigenicity of flagella associated proteins. ${ }^{53}$

Step 3: adhesin-mediated attachment to host receptors: Adhesins are virulence proteins that enable bacteria to bind cells in the host. Although many dangerous bacteria produce adhesins of varied types, they are frequently encoded on the bacterial backbone DNA. ${ }^{54} H$. pylori adheres to mucins by using adhesion molecules or other molecules on its surface, enabling the bacteria to infiltrate the gastric mucosa epithelium. This event causes the activation of various bacterial genes, including some that code for virulence factors and protect the pathogen against clearance mechanisms including liquid flow, peristaltic movement, or mucous layer shedding. ${ }^{55} \mathrm{H}$. pylori adhesion to the mucus layer of the gastric epithelium is critical for the bacteria for initial colonization and persistence in the human stomach over decades or perhaps a lifetime. When $H$. pylori colonizes the stomach, it triggers humoral and cellular immune responses that seldom result in bac-

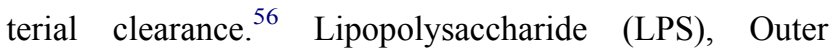
Membrane Proteins, blood group antigen-binding adhesin A (Baba), sialic acid-binding adhesin (SABA), adherence associated lipoproteins, outer inflammatory protein A, HOMB, and HopZ are all Helicobacter pylori adhesionrelated molecules. ${ }^{57} \mathrm{HomB}$ is an antigenic protein found in the membrane of $H$. pylori. Furthermore, the protein is linked to IL- 8 release in vitro and contributes to bacterial adhesion, both of which are linked to the amount of HomB copies in a strain ${ }^{42}$ Housekeeping genes, virulence genes, lipopolysaccharide (LPS), and several OMP producing genes are all affected by $H$. pylori genetic diversity, which contributes to host adaptation, persistence, and immune response evasion, resulting in chronic inflammatory reactions. The involvement of numerous Lewis-like antigens and adhesins factors has recently progressed for interaction of host cell. These interactions are described in terms of bacterial colonization and pathogenicity. ${ }^{57}$

Step 4: Toxin and host tissue damage: Ammonia (urease), lysolecithin (phospholipases), and acetaldehyde are all harmful chemicals produced by Helicobacter pylori enzymes (alcohol dehydrogenase). Ammonia's detrimental effects have been examined the most, and it appears to be a potential pathogenicity mechanism. ${ }^{58}$ CytotoxinAssociated Gene A (CagA): CagA is one of the pathogenicity factors of the bacterial pathogen Helicobacter pylori that has been extensively explored. The $H$. pylori cag-Type IV secretion mechanism is used to inject it into host cells. CagA is characterized as an oncogenic protein because of its link to gastric cancer. ${ }^{59}$

Vacuolating cytotoxin A (VacA): VacA is diffusible, pore-forming, exotoxin secreted by Helicobacter pylori, is epidemiologically connected to gastrointestinal illness in humans. ${ }^{60}$ Additionally, VacA is a multi-functional toxin that can produce "vacuoles," which resemble late endosomes and early lysosomes; be taken up by the cell and localized to the mitochondria, causing apoptosis; bind to a protein on the cell membrane, causing inflammation; and obstruct T-cell activation and proliferation. ${ }^{61}$ Dur VacA is a multi-functional toxin that can produce "vacuoles," which resemble late endosomes and early lysosomes; be taken up by the cell and localized to the mitochondria, causing apoptosis; bind to a protein on the cell membrane, causing inflammation; and obstruct T-cell activation and proliferation. Cytochrome $\mathrm{C}$ is released from the mitochondrial intermembrane gap into the cytoplasm via an unknown process, which activates downstream executioner caspases, resulting in cell death. It has been postulated that VacA can produce membrane-embedded pores at the inner-mitochondrial membrane, resulting in the dissipation of the mitochondrial electrochemical membrane potential, in accordance with its proposed pore-forming capabilities. $^{61}$

In general: Urease-dependent ammonia production elevates the $\mathrm{pH}$ during initial infection of the stomach lumen, which enhances bacterial survival and solubilizes the mucous gel to assist bacterial motility. Helical rod form and chemotaxis enhance flagellar movement away from 
the acidic lumen to Helicobacter pylori's preferred habitat, which lies on and close to gastric epithelial cells. SabA, BabA, and other adhesins factors may alter the balance of bacteria from mucus-associated to cell-associated bacteria $^{51}$ Vacuolating cytotoxin (VacA), cytotoxinassociated gene A (CagA), and CagL, all of which have diverse cellular targets, influence gastric epithelial cell behaviour. The RGD motif of CagL mediates interactions with the 51-cell surface receptor, whereas interactions with the other cell surface receptor (v5 integrin) are RGDindependent. $^{62}$ The combined action of these three effectors causes a number of changes in the gastric epithelial cell, including CagA and VacA disruption of cell polarity, which can promote iron acquisition and cell extrusion; CagA- and CagL-dependent induction of chemokines and/or the gastric hormone gastrin; and CagL-dependent inhibition of acid secretion by the $(\mathrm{H}++\mathrm{K}+)$ ATPase and cellular proliferation. ${ }^{63} \mathrm{CagA}$ and $\mathrm{CagY}$ have been proven to bind 51 integrins, however the exact interaction surface is unknown. PS stands for phosphatidylserine, and T4SS stands for type IV secretion system. ${ }^{64}$

\section{Giardia lamblia}

Giardia intestinalis can damage enterocytes and the loss of the brush border of epithelial cells in the intestine, resulting in microvilli shortening and impaired epithelial barrier function. Aqueous diarrhea, steatorrhea, nausea, abdominal pain, vomiting, and weight loss are all symptoms of this condition. Enhanced pro-apoptotic mechanisms, following breakdown of the intestinal epithelial barrier, hypersecretion of electrolytes, and increased exposure to luminal antigens by subepithelial host immune cells are hypothesized to be involved in Giardia pathogenesis. ${ }^{65}$ Giardia infects surrounding tissues and entering the bloodstream without passing the epithelium and cause illness. The epithelial abnormalities that cause intestinal malabsorption and diarrhea in giardiasis appear to be comparable to those seen in other enteric disorders like bacterial enteritis, chronic food allergy, Crohn's disease, and celiac disease. ${ }^{66}$ The mechanisms responsible for pathophysiology in giardiasis include both parasite and host immune factors. G. lamblia virulence factors have been suggested on the basis of their targeted effects. ${ }^{16}$ Giardiasis is a parasitic infection caused by the Giardia parasite. In humans, the parasite clings to the lining of the small intestine, causing diarrhea and preventing the body from absorbing fats and carbohydrates from digested foods. ${ }^{67}$ An illness causes a person's or an organ's function to be disrupted. A pathophysiologic alteration, for example, is a change in function as opposed to a structural flaw. The four pairs of flagella allow for movement, while the ventral sticky disc proteins and surface lectins promote proper attachment to upper small intestine enterocytes, while the four pairs of flagella allow for mobility and assist elude host IgA directed clearance. ${ }^{68}$

The parasite's arginine deiminase depletes arginine in intestinal epithelial cells, limiting intestinal epithelial proliferation and nitric oxide generation ${ }^{69}$ and The degradation of chemokine ligand 8 (CXCL8) by cathepsin B-like proteases results in reduced neutrophil chemotaxis. In a myosin light chain kinase-dependent way, Giardia also causes enterocyte apoptosis and destroys the epithelial barrier. Maldigestion and malabsorption result from CD8 + lymphocyte-dependent microvillus shortening. Indeed, when compared to controls, the height of microvilli as well as digestive enzyme activity did not differ following Giardia infection in immune defective mice unable to manufacture $\mathrm{T}$ cells. Together, these findings show that parasites and host variables both play a role in giardiasis epithelial abnormalities. Giardia may exacerbate these symptoms by increasing chloride output and intestinal transit, all of which lead to diarrhea. In some situations, Giardia infection can cause bile hypersecretion, which can lead to steatorrhea when combined with lipid malabsorption. ${ }^{15,45-47}$ In general giardiasis is multifactorial disease, reflecting the complex interplay between the host and parasite.

(1) Diffuse shortening of microvilli and inhibition of brush border enzymes. This involves attachment of the parasites and CD8+ T cells. (2) Induction of chemokines in IECs, resulting in attraction of immune cells like mast cells and dendritic cells (DC). (3) Disruption of tight junctions. (4) Induction of apoptosis. (5) Starvation for arginine in IECs, resulting in less NO, cell cycle arrest and apoptosis. (6) Increased intestinal permeability induced by mechanisms three to five. (7) Increased chloride ion secretion. (8) Intestinal hypermotility. (9) Crypt hyperplasia and increased mucus secretion. (10) Changed composition of bacterial normal flora. The parasite and host cells secrete specific proteins during the interaction (ESPs).

\section{Virulence Factors and Antigenic Variation of G. lamblia}

By developing into infective cysts, giardia trophozoites undergo fundamental alterations in order to survive 
outside of their host's intestine. Cyst wall components are synthesized, processed, transported, secreted, and assembled extracellularly during encystation. Giardia undergoes antigenic variation to survive within the gut, a process in which the parasite swaps its main surface molecules on a regular basis, allowing it to dodge the host's immune response and cause chronic and repeated infections. ${ }^{70}$ Cathepsin $\mathrm{B}$ is a known virulence factor and secreted protein that inhibits neutrophil chemotaxis by degrading IL-8. Cathepsin B and cystatin, a protease inhibitor of cathepsins, were upregulated in response to secreted host factors. ${ }^{71}$ Controlling giardia infections necessitates both innate and adaptive immune responses. Specific antibody production is linked to parasite clearance, and hypogammaglobulinemia patients have a greater rate of symptomatic infections. Antibodies are required for clearance late in infection in mice, with secretory immunoglobulin A being the most critical antibody isotype. Antigenic variation is a technique by which protozoan parasites evade the humoral immune response of their vertebrate hosts. ${ }^{16}$ Antigenic variation permits the pathogen to not only escape the immune response of its present host, but also to infect previously infected hosts again. Immunity to re-infection is based on the acquired immune response's detection of the pathogen's antigens, which are "remembered". 72 Antigenic variation occurs in Giardia lamblia both in vitro and in the intestines of infected people. The principal antigens identified by the host are variant-specific surface proteins (VSPs), which cover the whole surface of the trophozoites. Significant progress has been made in understanding the antigen switching process in the last year, indicating that antigenic variation in Giardia is regulated at the post-transcriptional level by a mechanism comparable to RNA interference (RNAi). ${ }^{73}$ VSP mRNA silencing and/or translational repression are mediated by several RNAi pathway enzymes. Although there are still some unanswered concerns about how individual VSP antigens are chosen for expression on the parasite surface, it is obvious that an epigenetic mechanism is at work. ${ }^{74}$

\section{Secreted Cysteine Protease Expression and Colonization of $G$. lamblia}

Enzymes that break down cysteine are known as cysteine proteases. Cysteine proteases are enzymes that have a cysteine thiol residue in their catalytic core (CPs). Clan $\mathrm{CA}$, which comprises papain- and calpain-like peptidases, and clan $\mathrm{CD}$, which contains calpain-like peptidases, make up the majority of parasite-produced CPs. The clan CA proteases, which represent for $84 \%$ of parasite CPs, are divided into 24 families based on their primary sequence and tertiary structure. The family $\mathrm{C} 1$ (eg, C1A), which accounts for $45 \%$ of protozoan peptidases, is split into two cysteine cathepsin subfamilies, catB-like and cathepsin L-like (EC. 3.4.22.15) enzymes, based on pro-domain sequences and length. ${ }^{75,76}$ Cysteine proteases (CPs) are recognized as virulence factors in Giardia, their exact role in the disease's molecular pathogenesis remains unknown. The goal of this study was to describe the three primary secreted CPs (CP14019, CP16160, and CP16779), which were discovered in the medium during interaction with intestinal epithelial cells (IECs) in vitro using mass spectrometry. The CPs were first epitope-tagged and found in endoplasmic reticulum and cytoplasmic vesicle-like structures. Second, we demonstrated that recombinant CPs produced in Pichia pastoris are more active in an acidic environment (pH 5.5-6) and used fluorogenic substrates to assess the kinetic characteristics. ${ }^{77,78}$ Giardia CP activity plays a role in trophozoite attachment to IECs. Taken together, our findings show that Giardia CPs play a significant role in Giardiaintestinal colonization interactions. ${ }^{79}$ Giardia excretorysecretory products such as surface proteins, secreted proteases, and extracellular vesicles appear to play critical roles in Giardia infection pathogenesis, compromising host epithelial integrity. Macrovesicles (MVs) are secreted by Giardia, and they may play a role in innate immune system activation, host attachment, and toxicity. ${ }^{77,80} \mathrm{CP}$ is also extensively produced during the trophozoite and encystation phases. CWPs, such as CPW2, are expressed during encystation alongside intracellular CP2, which processes CWP2 to initiate polymerization and cyst wall formation. ${ }^{75}$ Giardia CPs disrupt epithelial apical junctional complexes in Giardiasis, resulting in impaired barrier function as a pathophysiological process. Several studies have shown that Giardia causes reductions in IEC transepithelial resistance (TER), a marker of epithelial integrity and permeability. ${ }^{75,81} \mathrm{CP} 2$ causes a variety of epithelium injury in the host. Induce apoptosis and villin degradation in epithelial cells. Recombinant CP2 induces the formation of apoptotic bodies in epithelial cells. Phosphatidylserine levels rise in response to $\mathrm{CP} 2$, as does caspase-3 synthesis, which is time-dependent, and PARP-1 cleavage. Furthermore, the CP inhibitor E-64 against CP2 inhibited the development of apoptosis in IEC-6 cells, demonstrating that CPs play a role in Giardia-induced cell death. ${ }^{81}$ Giardia Secreted cysteine 
protease promotes the secretion of antimicrobial peptides such as beta-defensin 2 and is important for intestinal mucosal homeostasis and antimicrobial defense against entomopathogens such as Helicobacter pylori. Human -defensin-2 (HBD-2) is induced in IECs in vitro after exposure to stomach epithelial cells during human Helicobacter pylori infection, for example. ${ }^{82}$

\section{Coinfection}

$H$. pylori and $G$. lamblia coinfection in stomach causes duodenal biopsies. H. pylori play a role for $G$. lamblia colonization by different mechanisms. H. pylori producing urease, which converts urea from the stomach wall to ammonia, raising the stomach $\mathrm{pH}$ and allowing intestinal parasites to readily pass and reach the intestine. ${ }^{83,84}$ Parasites, on the other hand, escape the immune response by expressing antigenic variation on their surface or employing other unique mechanisms such as nutrition deprivation. The survival strategies used by Giardia lamblia after infection are examined in this paper. ${ }^{85}$ Levels of IL-18 in gastric epithelial cells and the monocyte cell line THP-1 increased when cocultured with Helicobacter pylori. H. pylori-infected epithelial cells and monocytes produced significantly more IL-18 in both systems. The intensity of gastric inflammation was closely linked with IL-18 levels in $H$. pylori-infected gastric mucosa, demonstrating that H. pylori-induced IL-18 plays a significant role in gastric damage. ${ }^{86}$ Furthermore, decreased acid secretion may result in the generation of pro-inflammatory cytokines such as IL$1 \mathrm{~b}$, a powerful inhibitor of gastric acid production, and a secreted $H$. pylori component that may directly target parietal cells. This creates an ideal environment for $G$. lamblia to reproduce in the duodenum, where the trophozoites bind to enterocytes, causing damage and generating gastrointestinal symptoms. ${ }^{5,87}$

The colonization of stomach mucosa by $H$. pylori generation of acute and subsequently chronic inflammation, as well as the emission of toxins and enzymes by the bacteria which resulted in disruption epithelial cells. ${ }^{88}$ Gastric atrophy and intestinal metaplasia are also one of the complications of $H$. pylori infection that may also facilitate Giardia colonization of the stomach. ${ }^{89}$ Even though the mechanisms are different both organisms have dual or synergistic effect on the host by inducing epithelial cell damage, destructing the mucus and tight junction, gastro intestinal metaplasia and gastritis resulting from each organism predisposes the person to the other. The coinfection rate of H. pylori and G. lamblia coinfection as shown (Table 1).

Table I Coinfection Rate of $H$. pylori and Intestinal Parasites

\begin{tabular}{|l|l|l|}
\hline Number of Study Subject & Prevalence and Coinfection Rate of Infectious Agent & Country (Reference) \\
\hline 318 & $70.8 \%$ & Venezuela $^{90}$ \\
\hline 427 & $30.2 \%$ & Uganda $^{17}$ \\
\hline 801 & $52.5 \%$ & Egypt $^{91}$ \\
\hline 120 & $50 \%$ & Mexico $^{92}$ \\
\hline 188 & $54.5 \%$ & Mexico $^{92}$ \\
\hline 215 & $45 \%$ & Colombia $^{93}$ \\
\hline 155 & $21 \%$ & Colombia $^{93}$ \\
\hline 68 & $29.7 \%$ & Iran $^{30}$ \\
\hline 206 & $51.4 \%$ & Egypt $^{94}$ \\
\hline 98 & $45.8 \%$ & Turkey $^{5}$ \\
\hline 187 & $8 \%$ & Pakistan $^{95}$ \\
\hline 100 & $5 \%$ & Sudan $^{96}$ \\
\hline 363 & $26.3 \%$ & Ethiopia $^{20}$ \\
\hline 434 & $13.1 \%$ & Ethiopia $^{97}$ \\
\hline
\end{tabular}




\section{Immunological Mechanisms}

The Immune Response to Helicobacter pylori are both humoral and cellular immune responses are induced by H. pylori. IgA, IgM, and IgG isotypes have been demonstrated in both local and systemic antibody responses H. pylori infection also induces the activation of innate and acquired response from the host immune system. ${ }^{98}$ Despite powerful innate and adaptive immune responses, H. pylori survives in the stomach mucosa for a long time. In addition to virulence factors, the ability of $H$. pylori to escape, disrupt, and manipulate the host immune system has a significant impact on its survival. By changing its surface components, this bacterium can avoid being recognized by innate immune receptors. In addition, H. pylori subverts adaptive immunity by modulating effector T cells. ${ }^{99}$ Th1 and Th2 cells both have a role in defining this response, with Th1 taking the lead. These immune cells regulate the cellular response of the host, increase the release of proinflammatory cytokines such as IL-2 and INF-, and suppress Th2-dependent immunological activity Th1 and Th2 cells both have a role in defining this response, with Th1 taking the lead. These immune cells regulate the cellular response of the host, increase the release of proinflammatory cytokines such as IL-2 and INF-, and suppress Th2-dependent immunological activity. Th17 cells, which are the source of the cytokine IL-17, are also recruited. ${ }^{100}$ The production of IL-8, a chemoattractant pro-inflammatory cytokine that stimulates neutrophil chemotaxis into stomach tissue, is affected by an increase in IL-17 release. Chronic neutrophil infiltration wreaks havoc on the mucosa because these cells produce oxidative stress from reactive oxygen and nitrogen. ${ }^{101}$ Despite the fact that both Th1 and Th17 cells are involved in the antimicrobial response to H. pylori, neither is capable of entirely eliminating the bacteria from the body. This is because $H$. pylori encourages the recruitment of regulatory lymphocytes (Treg), which suppresses the antibacterial host immune response and prolongs the infection of the gastric mucosa. ${ }^{21}$ H. pylori manipulates host immune responses as an effective technique in the development of gastrointestinal illnesses, in addition to other virulence factors that lead to its survival. The mucus released by epithelial cells and the innate immune cells in the lamina propria are the principal defenses against H.pylori. ${ }^{99}$

Giardia lamblia infection elicits both innate and adaptive immune responses in humans and animals. The innate immune system is responsible for the first identification of Giardia by the host. Antimicrobial peptides like as defensins are released by Paneth cells in the Lieberkühn crypts in addition to pancreatic secretions. Lactoferrin, which has been demonstrated to be harmful to Giardia trophozoites in vitro, is released continually by the gallbladder. ${ }^{102}$ The epithelial cells lining the wall of the intestine respond to Giardia trophozoite contact by production of cytokines and chemokines. Dendritic cells are also able to recognize and respond to Giardia trophozoite and nitric oxide could inhibit trophozoites replication. ${ }^{96}$

The adaptive system establishes specific response and memory towards a pathogen and is responsible for antibody production. Infection with $G$. lamblia typically results in strong antibody response against the parasite. ${ }^{103}$ For instance, incubation of trophozoites with specific antibody can lead to inhibition of parasites replication as well as parasite death depending on the particular antibodies and the presence of complement. Immunoglobulin A (IgA) is believed to be more important in parasite control and also passive transfer of anti-Giardia antibodies in milk can help protect human new born. ${ }^{104}$ Conversely, variant specific surface proteins (VSPs) coat the surface of Giardia trophozoite may contribute to pathogenicity and allow Giardia to evade immunological memory responses. ${ }^{105}$

T cells has crucial role to play a key part in the immune response to Giardia infection, and memory responses to the parasite can endure for years. Three animal investigations of Giardia infection have recently discovered TH17 responses. ${ }^{106}$ In CD4+ effector memory (EM) T cells, the production of tumor necrosis factor alpha (TNF-), gamma interferon, interleukin-17A (IL-17A), IL-10, and IL-4 was evaluated. It is unknown how important the cytokines IL$17 \mathrm{~A}, \mathrm{IFN}-$, TNF-, IL-4, and IL-10 are in the T cell response to Giardia infection in humans. ${ }^{107}$

T-cells, which include CD4+ helper T cells and CD8+ or cytotoxic $\mathrm{T}$ cells, are another component of the adaptive immune response that is necessary for appropriate Giardia infection treatment (Tc). CD4+ helper T cells, in addition to supporting Tc cell activation and B lymphocyte growth, are primarily responsible for this protective effect. ${ }^{108}$ Mast cell activation causes increased smooth muscle contractions, which affects intestinal motility. CD8+ cells produce a robust immunological response (pathophysiology) that results in diffuse microvilli shortening. IL-17 has been shown to play a role in Giardia infections in both mice and humans. ${ }^{96}$ 
The presence of H. pylori and G. lamblia in the same environment can have a major impact on the host immunological response. Giardiasis is caused by an increase in intraepithelial lymphocyte infiltration caused by $H$. pylori, which leads in epithelial damage and malfunction. As a result, G. lamblia causes a wide loss of the microvillous brush border, resulting in disaccharidase deficiency and malabsorption of electrolytes, minerals, and water, producing diarrhea and crypt hyperplasia. ${ }^{109}$

Similarly, the cohabitation of G. lamblia and H. pylori in the host organism produces an immune response marked by lymphocyte polarization toward Th1. These immune cells are more numerous in the stomach of Helicobacter pylori-infected individuals, causing inflammation. The antimicrobial type 1 response is characterized by the release of pro-inflammatory cytokines such as IL 2, IL-12, and IFN-. As a result of their coexistence, the host suffers tissue damage. In H. pylori infection, the type 1 immune response is also necessary for the development of gastritis, peptic ulcers, gastrointestinal metaplasia, and gastric cancer. ${ }^{110}$ For this reason, the presence of G. lamblia and $H$. pylori together significantly aggravate these ailments. ${ }^{111}$

\section{Clinical Features}

The bacteria H. pylori (Helicobacter pylori) can cause an infection in the stomach or duodenum (first part of the small intestine). Peptic ulcer disease is most commonly caused by this. The stomach lining can also be inflamed and irritated by $H$. pylori (gastritis). Long-term H. pylori infection can lead to stomach cancer if left untreated. ${ }^{35}$ H. pylori infection can last for years after colonization, and it can cause acute and chronic illnesses. Infections that go untreated can become chronic, even life-threatening. When a person experiences upper stomach pain, nausea, or heartburn, it is likely that they have gastroenteritis. Swelling of the tissue leading into the small intestine can be caused by gastric ulcers. ${ }^{112}$ This makes it difficult for food to pass through the stomach, resulting in pain, bloating, nausea, and vomiting after eating. Peptic ulcers are another consequence that can result in perforation, bleeding, and blockage. H. pylori is also a risk factor for gastric cancer, according to several research. ${ }^{113}$

G. lamblia is a non-invasive bacterium that reproduces by attaching itself to the intestinal epithelium. Giardiasis is a disease with varying degrees of severity; it can be acute or chronic, and asymptomatic carriers are prevalent. ${ }^{114}$ Symptoms of disease usually appear 7-12 days after infection. Diarrhea, nausea, vomiting, weight loss, malabsorption, and steatorrhea (fatty stools) are the most prevalent clinical indications of infection. ${ }^{105}$ It also causes vitamin deficiencies, especially in the fat-soluble vitamins $\mathrm{A}, \mathrm{D}, \mathrm{E}$, and $\mathrm{K}$, as well as folic acid, which can lead to a variety of health issues. Some individuals have a slight ailment that goes away on its own, while others may have a serious disease that lasts a long time. Achlorhydria can lead to symptomatic giardia infection, hypogammaglobulinemia, and a lack of secretory IgA in the small intestine (John and Petri, 2006).

Upper gastrointestinal problems caused by both organisms include upper abdomen pain, bloating, nausea, vomiting, early satiation, epigastric pain/burning, and belching. Acute $H$. pylori infection is followed by hypochlorhydria, which makes it easier to acquire G. lamblia due to the loss of the gastric acid barrier, resulting in diarrhea and iron deficiency anemia (IDA). The ensuing synergistic effect of diarrheal illness and micronutrient deficiencies on child growth and cognitive function has major public health implications for these countries' socioeconomic development. ${ }^{115}$ Differentiation by physical examination is impossible in people who are coinfected with those diseases. As a result, laboratory testing is advised. Additionally, triple therapy (clarithromycin + amoxicillin or metronidazole plus a proton pump inhibitor like omeprazole or lansoprazole) is required). ${ }^{105}$

\section{Conclusion and Recommendations}

G. lamblia and $H$. pylori coinfection can be caused by common risk factors and transmission routes. They take possession of the upper gastrointestinal tract and activating predictable side effects on the gastrointestinal tract. H. pylori induced-pathologies and hypochlorhydria in the stomach could facilitate Giardia colonization in gastric mucosa. The presences of both pathogens together significantly influence the modulation of the host immune response. H. pylori induce $\mathrm{CD} 8^{+} \mathrm{T}$ cell by increase infiltration of intraepithelial lymphocytes that ultimately lead to epithelial injury and malfunction in giardiasis. Th1 and Th2 cells both have a role in defining this response. In the same manner the presence of G. lamblia with H. pylori in the host organism results immune response with strong polarization of lymphocytes towards Th1 and leads to host damage. So, their presence together aggravates host tissue damage.

Even though the mechanisms are different both organisms have dual or synergistic effect on the host by inducing epithelial cell damage, destructing the mucus and 
tight junction, gastro intestinal metaplasia and gastritis Both organisms have similar clinical manifestations. Thus, differentiation solely by clinical sign and symptoms is not possible for patients coinfected with those pathogens. Therefore, laboratory diagnosis is essential for the effective treatment and management of infections caused by $G$. lamblia and $H$. pylori. The biological mechanisms behind this potential microbial interplay indeed should be investigated more by researchers. Since both pathogens can cultured in vitro and in vivo assays may be implemented to gain further understanding of how they interact with the host's immune response. There should be also improvement of health measures such as personal hygiene and water purification are useful ways for elimination of the infections.

\section{Abbreviations}

VacA, Vacuolating cytotoxin A; cagA, cytotoxinassociated gene; PAI, the cag phosphoribosyl anthranilate isomerase; CP, Immunoglobulin, cysteine protease; IEC, intestinal epithelial cells.

\section{Author Contributions}

All authors contributed significantly to the conception, article writing and revision as well as decisions to the journal to which the article would be submitted, gave final approval to the version for publication, and agreed to be accountable for all aspects of the work.

\section{Funding}

This study did not receive any funding in any form.

\section{Disclosure}

The authors report no conflicts of interest.

\section{References}

1. Noto JM, Peek RM. The Helicobacter pylori cag pathogenicity island. Helicobacter Species. Methods Mol Biol. 2012;921:41-50.

2. Backert S, Selbach M. Role of type IV secretion in Helicobacter pylori pathogenesis. Cell Microbiol. 2008;10(8):1573-1581. doi:10.1111/ j.1462-5822.2008.01156.x

3. Hatakeyama M. SagA of CagA in Helicobacter pylori pathogenesis. Curr Opin Microbiol. 2008;11(1):30-37. doi:10.1016/j.mib.2007. 12.003

4. Hatakeyama M. The role of Helicobacter pylori CagA in gastric carcinogenesis. Int $J$ Hematol. 2006;84(4):301-308. doi:10.1532/IJH 97.06166

5. Moreira ED, Nassri VB, Santos RS, et al. Association of Helicobacter pylori infection and giardiasis: results from a study of surrogate markers for fecal exposure among children. World $J$ Gastroenterol. 2005;11(18):2759. doi:10.3748/wjg.v11.i18.2759
6. Ryan U, Cacciò SM. Zoonotic potential of Giardia. Int J Parasitol. 2013;43(12-13):943-956. doi:10.1016/j.ijpara.20 13.06.001

7. Feng Y, Xiao L. Zoonotic potential and molecular epidemiology of Giardia species and giardiasis. Clin Microbiol Rev. 2011;24 (1):110-140. doi:10.1128/CMR.00033-10

8. Savioli L, Smith H, Thompson A. Giardia and Cryptosporidium join the 'neglected diseases initiative'. Trends Parasitol. 2006;22 (5):203-208. doi:10.1016/j.pt.2006.02.015

9. Isaeva G, Efimova N. Gastrointestinal giardiasis associated with Helicobacter pylori. Eksperimental'naia I Klinicheskaia Gastroenterologiia. 2010;47(6):30-34.

10. Rahman M, Sobur M, Islam M, et al. Zoonotic diseases: etiology, impact, and control. Microorganisms. 2020;8(9):1405. doi:10. 3390/microorganisms 8091405

11. Birrie H, Erko B. Giardiasis in Ethiopia. Ethiop J Health Dev. 1995;9(1):77-80.

12. Nkrumah B, Nguah SB. Giardia lamblia: a major parasitic cause of childhood diarrhoea in patients attending a district hospital in Ghana. Parasit Vectors. 2011;4(1):163. doi:10.1186/1756-33054-163

13. Katz DE, Taylor DN. Parasitic infections of the gastrointestinal tract. Gastroenterol Clin. 2001;30(3):797-815. doi:10.1016/ S0889-8553(05)70211-9

14. Cotton JA, Amat CB, Buret AG. Disruptions of host immunity and inflammation by Giardia duodenalis: potential consequences for co-infections in the gastro-intestinal tract. Pathogens. 2015;4 (4):764-792. doi:10.3390/pathogens 4040764

15. Buret AG, Amat CB, Manko A, et al. Giardia duodenalis: new research developments in pathophysiology, pathogenesis, and virulence factors. Curr Trop Med Rep. 2015;2(3):110-118. doi:10.1007/s40475-015-0049-8

16. Ankarklev J, Jerlström-Hultqvist J, Ringqvist E, Troell K, Svärd SG. Behind the smile: cell biology and disease mechanisms of Giardia species. Nat Rev Microbiol. 2010;8(6):413-422. doi:10.1038/nrmicro2317

17. Ankarklev J, Hestvik E, Lebbad M, et al. Common coinfections of Giardia intestinalis and Helicobacter pylori in non-symptomatic Ugandan children. PLoS Negl Trop Dis. 2012;6:e1780. doi:10.1371/journal.pntd.0001780

18. Misra V, Misra S, Dwivedi M, Singh P. Giardia lamblia trophozoites in gastric biopsies. Indian J Pathol Microbiol. 2006;49 (4):519-523.

19. Khalifa MM, Sharaf RR, Aziz RK. Helicobacter pylori: a poor man's gut pathogen? Gut Pathog. 2010;2(1):2. doi:10.1186/17574749-2-2

20. Seid A, Tamir Z, Kasanew B, Senbetay M. Co-infection of intestinal parasites and Helicobacter pylori among upper gastrointestinal symptomatic adult patients attending Mekanesalem Hospital, northeast Ethiopia. BMC Res Notes. 2018;11(1):1-6. doi:10.1186/s13104-018-3246-4

21. Larussa T, Leone I, Suraci E, Imeneo M, Luzza F. Helicobacter pylori and $\mathrm{T}$ helper cells: mechanisms of immune escape and tolerance. J Immunol Res. 2015;2015:1-10. doi:10.1155/2015/ 981328

22. Krzyżek P, Gościniak G. Frequency and immunological consequences of Helicobacter pylori and intestinal parasite co-infections: a brief review. Ann Parasitol. 2017;63(4): 255-263. doi:10.17420/ap6304.112

23. Ridley JW. Parasitology for Medical and Clinical Laboratory Professionals. Cengage Learning; 2012.

24. Hestvik E, Tylleskar T, Kaddu-Mulindwa DH, et al. Helicobacter pylori in apparently healthy children aged $0-12$ years in urban Kampala, Uganda: a community-based cross sectional survey. BMC Gastroenterol. 2010;10(1):62. doi:10.1186/1471-230X-1062 
25. Fouad SA, Esmat S, Basyoni MM, Farhan MS, Kobaisi MH. Molecular identification of Giardia intestinalis in patients with dyspepsia. Digestion. 2014;90(1):63-71. doi:10.1159/000362644

26. Olokoba A, Gashau W, Bwala S, Adamu A, Salawu F. Helicobacter pylori infection in Nigerians with dyspepsia. Ghana Med J. 2013;47(2):79-81.

27. Ivanov A. Giardia and giardiasis. Bulgar J Vet Med. 2010;13 (2):65-80.

28. Yakoob J, Jafri W, Abid S, et al. Giardiasis in patients with dyspeptic symptoms. World J Gastroenterol. 2005;11(42):6667. doi:10.3748/wjg.v11.i42.6667

29. Al-Mendalawi MD. Prevalence of Helicobacter pylori and parasites in symptomatic children examined for Helicobacter pylori antibodies, antigens, and parasites in Yemen. Saudi Med J. 2015;36(1):126. doi:10.15537/smj.2015.1.10871

30. Kazemian H, Shavalipour A, Mohebi R, et al. Estimation of the parasitic infection prevalence in children with Helicobacter pylori infection in Ilam city (2012-2013). Archiv Pediatr Infect Dis. 2014;2(3). doi:10.5812/pedinfect.15294

31. Zeyrek D, Zeyrek F, Cakmak A, Cekin A. Association of Helicobacter pylori and giardiasis in children with recurrent abdominal pain. Turkiye Parazitol Derg. 2008;32(1):4-7.

32. Peek RM, Blaser MJ. Helicobacter pylori and gastrointestinal tract adenocarcinomas. Nat Rev Cancer. 2002;2(1):28-37. doi:10.1038/nrc703

33. Sainsus N, Cattori V, Lepadatu C, Hofmann-Lehmann R. Liquid culture medium for the rapid cultivation of Helicobacter pylori from biopsy specimens. Eur J Clin Microbiol Infect Dis. 2008;27 (12):1209-1217. doi:10.1007/s10096-008-0567-6

34. Conteduca V, Sansonno D, Lauletta G, Russi S, Ingravallo G, Dammacco F. H. pylori infection and gastric cancer: state of the art. Int J Oncol. 2013;42(1):5-18. doi:10.3892/ijo.2012.1701

35. McColl KE. Helicobacter pylori infection. $N$ Engl $J$ Med. 2010;362(17):1597-1604. doi:10.1056/NEJMcp1001110

36. Kusters JG, Van Vliet AH, Kuipers EJ. Pathogenesis of Helicobacter pylori infection. Clin Microbiol Rev. 2006;19 (3):449-490. doi:10.1128/CMR.00054-05

37. Baj J, Forma A, Sitarz M, et al. Helicobacter pylori virulence factorsmechanisms of bacterial pathogenicity in the gastric microenvironment. Cells. 2021;10(1):27. doi:10.3390/cells10010027

38. Ansari S, Yamaoka Y. Helicobacter pylori virulence factors exploiting gastric colonization and its pathogenicity. Toxins. 2019;11(11):677. doi:10.3390/toxins 11110677

39. Lertsethtakarn P, Ottemann KM, Hendrixson DR. Motility and chemotaxis in Campylobacter and Helicobacter. Annu Rev Microbiol. 2011;65:389-410. doi:10.1146/annurev-micro-090110-102908

40. Ding S-Z, Goldberg JB, Hatakeyama M. Helicobacter pylori infection, oncogenic pathways and epigenetic mechanisms in gastric carcinogenesis. Future Oncol. 2010;6(5):851-862. doi:10.2217/fon. 10.37

41. Knorr J, Ricci V, Hatakeyama M, Backert S. Classification of Helicobacter pylori virulence factors: is CagA a toxin or not? Trends Microbiol. 2019;27(9):731-738. doi:10.1016/j.tim.2019.04.010

42. Roesler BM, Rabelo-Gonçalves EM, Zeitune JM. Virulence factors of Helicobacter pylori: a review. Clin Med Insights Gastroenterol. 2014;7:S13760.

43. Amieva MR, El-Omar EM. Host-bacterial interactions in Helicobacter pylori infection. Gastroenterology. 2008;134 (1):306-323. doi:10.1053/j.gastro.2007.11.009

44. Backert S, Clyne M. Pathogenesis of Helicobacter pylori infection. Helicobacter. 2011;16:19-25. doi:10.1111/j.15235378.2011.00876.x

45. Skoog EC, Deck SL, Entwistle HD, Hansen LM, Solnick JV. Characterization of the Cag pathogenicity island in Helicobacter pylori from naturally infected rhesus macaques. Microbiol Lett. 2016;363(24):fnw275. doi:10.1093/femsle/fnw275
46. Shiota S, Suzuki R, Yamaoka Y. The significance of virulence factors in H elicobacter pylori. J Dig Dis. 2013;14(7):341-349. doi:10.1111/1751-2980.12054

47. Talaiezadeh A, Hajiani E, Tarshizi MA. The relative frequency of the Helicobacter pylori infection in proximal gastric cancers. Pol Przegl Chir. 2013;85(11):657-662. doi:10.2478/pjs-2013-0099

48. Celli JP, Turner BS, Afdhal NH, et al. Helicobacter pylori moves through mucus by reducing mucin viscoelasticity. Proc Natl Acad Sci. 2009;106(34):14321-14326. doi:10.1073/pnas.0903438106

49. Kao C-Y, Sheu B-S, Wu -J-J. Helicobacter pylori infection: an overview of bacterial virulence factors and pathogenesis. Biomed J. 2016;39(1):14-23. doi:10.1016/j.bj.2015.06.002

50. Robinson K, Kenefeck R, Pidgeon EL, et al. Helicobacter pylori-induced peptic ulcer disease is associated with inadequate regulatory $\mathrm{T}$ cell responses. Gut. 2008;57(10):1375-1385. doi:10.1136/gut.2007.137539

51. Salama NR, Hartung ML, Müller A. Life in the human stomach: persistence strategies of the bacterial pathogen Helicobacter pylori. Nat Rev Microbiol. 2013;11(6):385-399. doi:10.1038/ nrmicro3016

52. Gu H. Role of flagella in the pathogenesis of Helicobacter pylori. Curr Microbiol. 2017;74(7):863-869. doi:10.1007/s00284-0171256-4

53. Skene C, Young A, Every A, Sutton P. Helicobacter pylori flagella: antigenic profile and protective immunity. FEMS Immunol Med Microbiol. 2007;50(2):249-256. doi:10.1111/j.1574695X.2007.00263.x

54. Gal-Mor O, Finlay BB. Pathogenicity islands: a molecular toolbox for bacterial virulence. Cell Microbiol. 2006;8(11):17 07-1719. doi:10.1111/j.1462-5822.2006.00794.x

55. Huang Y, Wang Q-L, Cheng D-D, Xu W-T, Lu N-H. Adhesion and invasion of gastric mucosa epithelial cells by Helicobacter pylori. Front Cell Infect Microbiol. 2016;6:159. doi:10.3389/ fcimb.2016.00159

56. Gupta N, Maurya S, Verma H, Verma VK. Unraveling the factors and mechanism involved in persistence: host-pathogen interactions in Helicobacter pylori. $J$ Cell Biochem. 2019;120 (11):18572-18587. doi:10.1002/jcb.29201

57. Oleastro M, Ménard A. The role of Helicobacter pylori outer membrane proteins in adherence and pathogenesis. Biology. 2013;2(3):1110-1134. doi:10.3390/biology2031110

58. Mégraud F. Toxic factors of Helicobacter pylori. Eur J Gastroenterol Hepatol. 1994;6:S5-10.

59. Jiménez-Soto LF, Haas R. The CagA toxin of Helicobacter pylori: abundant production but relatively low amount translocated. Sci Rep. 2016;6(1):1-7. doi:10.1038/srep23227

60. Holland RL, Bosi KD, Harpring GH, et al. Chronic in vivo exposure to Helicobacter pylori VacA: assessing the efficacy of automated and long-term intragastric toxin infusion. Sci Rep. 2020;10(1):1-16. doi:10.1038/s41598-020-65787-3

61. Palframan SL, Kwok T, Gabriel K. Vacuolating cytotoxin A (VacA), a key toxin for Helicobacter pylori pathogenesis. Front Cell Infect Microbiol. 2012;2:92. doi:10.3389/fcimb.2012.00092

62. Ansari S, Yamaoka Y. Helicobacter pylori virulence factor cytotoxin-associated Gene A (CagA)-mediated gastric pathogenicity. Int J Mol Sci. 2020;21(19):7430. doi:10.3390/ ijms 21197430

63. Pachathundikandi SK, Tegtmeyer N, Backert S. Signal transduction of Helicobacter pylori during interaction with host cell protein receptors of epithelial and immune cells. Gut Microbes. 2013;4(6):454-474. doi:10.4161/gmic.27001

64. Basso D, Plebani M, Kusters JG. Pathogenesis of Helicobacter pylori infection. Helicobacter. 2010;15:14-20. doi:10.1111/ j.1523-5378.2010.00781.x

65. Hawrelak J. Giardiasis: pathophysiology and management. Altern Med Rev. 2003;8(2):129-142. 
66. Buret AG. Mechanisms of epithelial dysfunction in giardiasis. Gut. 2007;56(3):316-317. doi:10.1136/gut.2006.107771

67. Minetti C, Chalmers RM, Beeching NJ, Probert C, Lamden K. Giardiasis. BMJ. 2016;355. doi:10.1136/bmj.i5369

68. Yu LZ, Birky CW, Adam RD. The two nuclei of Giardia each have complete copies of the genome and are partitioned equationally at cytokinesis. Eukaryotic Cell. 2002;1(2):191-199. doi:10.1128/EC.1.2.191-199.2002

69. Stadelmann B, Hanevik K, Andersson MK, Bruserud O, Svärd SG. The role of arginine and arginine-metabolizing enzymes during Giardia-host cell interactions in vitro. $B M C$ Microbiol. 2013;13(1):256. doi:10.1186/1471-2180-13-256

70. Docampo R, Lujan HD, Docampo R. Mechanisms of adaptation in the intestinal parasite Giardia lamblia. Essays Biochem. 2011;51:177-191. doi:10.1042/bse0510177

71. Emery SJ, Mirzaei M, Vuong D, et al. Induction of virulence factors in Giardia duodenalis independent of host attachment. Sci Rep. 2016;6(1):1-16. doi:10.1038/srep20765

72. Nash TE. Antigenic Variation in Giardia. Springer; 2011:245-257.

73. Prucca CG, Rivero FD, Luján HD. Regulation of antigenic variation in Giardia lamblia. Annu Rev Microbiol. 2011;65:611-630. doi:10.1146/annurev-micro-090110-102940

74. Martinez T, Wright N, Paneda C, Jimenez AI, Lopez-Fraga M. RNA interference-based therapeutics: harnessing the powers of nature. Drug Dev. 2011;265. doi:10.5772/29576

75. Allain T, Fekete E, Buret AG. Giardia cysteine proteases: the teeth behind the smile. Trends Parasitol. 2019;35(8):636-648. doi:10.1016/j.pt.2019.06.003

76. Alvarez VE, Iribarren PA, Niemirowicz GT, Cazzulo JJ. Update on relevant trypanosome peptidases: validated targets and future challenges. Biochimica et Biophysica Acta. 2020;1869:140577.

77. Ma'ayeh SY, Liu J, Peirasmaki D, et al. Characterization of the Giardia intestinalis secretome during interaction with human intestinal epithelial cells: the impact on host cells. PLoS Negl Trop Dis. 2017;11(12): e0006120. doi:10.1371/journal.pntd.0006120

78. Liu J, Ma'ayeh S, Peirasmaki D, Lundström-Stadelmann B, Hellman L, Svärd SG. Secreted Giardia intestinalis cysteine proteases disrupt intestinal epithelial cell junctional complexes and degrade chemokines. Virulence. 2018;9(1):879-894. doi:10.1080/ 21505594.2018.1451284

79. Li Z. Serglycin-dependent mast cell-specific proteases and their potential interactions with Giardia intestinalis. 2019.

80. Evans-Osses I, Reichembach LH, Ramirez MI. Exosomes or microvesicles? Two kinds of extracellular vesicles with different routes to modify protozoan-host cell interaction. Parasitol Res. 2015;114(10):3567-3575. doi:10.1007/s00436-015-4659-9

81. Ma'ayeh SY. Molecular and Cellular Interactions of Four Giardia Duodenalis Isolates with the Murine Intestinal Epithelial Cell Line IEC-6 in Vitro. Monash University; 2013.

82. Manko A, Motta J-P, Cotton JA, et al. Giardia co-infection promotes the secretion of antimicrobial peptides beta-defensin 2 and trefoil factor 3 and attenuates attaching and effacing bacteria-induced intestinal disease. PLoS One. 2017;12(6): e0178647. doi:10.1371/journal.pone.0178647

83. Wang F, Xia P, Wu F, et al. Helicobacter pylori VacA disrupts apical membrane-cytoskeletal interactions in gastric parietal cells. $J$ Biol Chem. 2008;283(39):26714-26725. doi:10.1074/jbc.M800527200

84. Angelov TA, Kovacheva-Slavova MD, Iliev HI, Valkov HY, Vladimirov BG. Helicobacter Pylori Infection. Gastritis-New Approaches and Treatments. IntechOpen; 2019.

85. Rópolo AA, Touz MC. A lesson in survival, by Giardia lamblia. Sci World J. 2010;10:2019-2031. doi:10.1100/tsw.2010.200

86. Yamauchi K, Choi I-J, Lu H, Ogiwara H, Graham DY, Yamaoka Y. Regulation of IL-18 in Helicobacter pylori infection. J Immunol. 2008;180(2):1207-1216. doi:10.4049/ jimmunol.180.2.1207
87. Lane S, Lloyd D. Current trends in research into the waterborne parasite Giardia. Crit Rev Microbiol. 2002;28(2):123-147. doi:10.1080/1040-840291046713

88. Dias A, Garcia C, Majewski M, et al. Gastric juice prostaglandins and peptide growth factors as potential markers of chronic atrophic gastritis, intestinal metaplasia and gastric cancer: their potential clinical implications based on this pilot study. Dig Dis Sci. 2011;56(11):3220-3225. doi:10.1007/s10620-011-1758-z

89. Liu Y, Vosmaer G, Tytgat G, Xiao S, Ten Kate F. Gastrin (G) cells and somatostatin (D) cells in patients with dyspeptic symptoms: Helicobacter pylori associated and non-associated gastritis. J Clin Pathol. 2005;58(9):927-931. doi:10.1136/jcp.2003.010710

90. Fuenmayor-Boscán AD, Hernández IM, Valero KJ, Paz AM, Sandrea LB, Rivero Z. Association between Helicobacter pylori and intestinal parasites in an Añu indigenous community of Venezuela. Indian $J$ Gastroenterol. 2016;35(2):106-112. doi:10.1007/s12664-016-0641-4

91. El-Badry AA, Ghieth MA, Ahmed DA, Ismail MA. Giardia intestinalis and helicobacter pylori co-infection: estimated risks and predictive factors in Egypt. J Egypt Soc Parasitol. 2017;47 (1):19-24. doi:10.21608/jesp.2017.77957

92. Torres J, Perez GP, Ximenez C, et al. The association of intestinal parasitosis and $\mathrm{H}$. pylori infection in children and adults from a Mexican community with high prevalence of parasitosis. Helicobacter. 2003;8(3):179-185. doi:10.1046/j.1523-5378.20 03.00142.x

93. Whary MT, Sundina N, Bravo LE, et al. Intestinal helminthiasis in Colombian children promotes a Th2 response to Helicobacter pylori: possible implications for gastric carcinogenesis. Cancer Epidemiol Prev Biomark. 2005;14(6):1464-1469. doi:10.1158/ 1055-9965.EPI-05-0095

94. Sabah AA, Gneidy MR, Saleh NMK. Prevalence of Helicobacter pylori infection among adult patients with different gastrointestinal parasites in Tanta City district. J Egypt Soc Parasitol. 2015;45(1):101-106. doi:10.21608/jesp.2015.89704

95. Hasan SM, Shabbir A, Shaikh A, Khan TN, Irshad Z. Endoscopic biopsy proven co-infection of Helicobacter pylori and Giardia lamblia in adult population of Karachi city. Pak J Med Dent. 2019;8(2):4.

96. Ahmed NFM. Prevalence Rate of Giardia Lamblia/Helicobacter Pylori Co-Infections in Khartoum State-Sudan. Sudan University of Science \& Technology; 2016.

97. Spotts H, Walelign S, Tesfaye M, Desta K, Tsegaye A, Taye B. Concurrent infection of intestinal parasites and Helicobacter pylori among school-age children in Central Ethiopia. Parasite Epidemiol Contr. 2020;11:e00177. doi:10.1016/j.parepi.2020. e00177

98. Ieni A, Barresi V, Rigoli L, Fedele F, Tuccari G, Caruso R Morphological and cellular features of innate immune reaction in Helicobacter pylori gastritis: a brief review. Int $J$ Mol Sci. 2016;17(1):109. doi:10.3390/ijms17010109

99. Karkhah A, Ebrahimpour S, Rostamtabar M, et al. Helicobacter pylori evasion strategies of the host innate and adaptive immune responses to survive and develop gastrointestinal diseases. Microbiol Res. 2019;218:49-57. doi:10.1016/j.micres.2018.09.011

100. Khamri W, Walker MM, Clark P, et al. Helicobacter pylori stimulates dendritic cells to induce interleukin-17 expression from CD4+ T lymphocytes. Infect Immun. 2010;78(2):845-853. doi:10. 1128/IAI.00524-09

101. Luzza F, Parrello T, Monteleone G, et al. Up-regulation of IL-17 is associated with bioactive IL-8 expression in Helicobacter pylori-infected human gastric mucosa. J Immunol. 2000;165 (9):5332-5337. doi:10.4049/jimmunol.165.9.5332

102. Brenchley JM, Douek DC. Microbial translocation across the GI tract. Annu Rev Immunol. 2012;30:149-173. doi:10.1146/annurevimmunol-020711-075001 
103. Fink MY, Singer SM. The intersection of immune responses, microbiota, and pathogenesis in giardiasis. Trends Parasitol. 2017;33(11):901-913. doi:10.1016/j.pt.20 17.08 .001

104. Evering T, Weiss L. The immunology of parasite infections in immunocompromised hosts. Parasite Immunol. 2006;28(11):549-565. doi:10.1111/j.1365-3024.20 06.00886.x

105. Bartelt LA, Sartor RB. Advances in understanding Giardia: determinants and mechanisms of chronic sequelae. F1000Prime Rep. 2015;7. doi:10.12703/P7-62

106. Hanevik K, Kristoffersen E, Svard S, et al. Human cellular immune response against Giardia lamblia 5 years after acute giardiasis. J Infect Dis. 2011;204(11):1779-1786. doi:10.1093/ infdis/jir639

107. Saghaug CS, Sørnes S, Peirasmaki D, Svärd S, Langeland N, Hanevik K. Human memory CD4+ T cell immune responses against Giardia lamblia. Clin Vaccine Immunol. 2015;23 (1):11-18. doi:10.1128/CVI.00419-15

108. Janeway CA. How the immune system works to protect the host from infection: a personal view. Proc Natl Acad Sci. 2001;98 (13):7461-7468. doi:10.1073/pnas.131202998
109. Buret A. Immunopathology of giardiasis: the role of lymphocytes in intestinal epithelial injury and malfunction. Memórias Do Instituto Oswaldo Cruz. 2005;100:185-190. doi:10.1590/S007402762005000900032

110. Houghton J, Fox JG, Wang TC. Gastric cancer: laboratory bench to clinic. J Gastroenterol Hepatol. 2002;17(4):495-502. doi:10.1046/j.1440-1746.2002.02770.x

111. Kasper LH, Buzoni-Gatel D, Portnoy DA. Ups and downs of mucosal cellular immunity against protozoan parasites. Infect Immun. 2001;69(1):1-8. doi:10.1128/IAI.69.1.1-8.2001

112. Andersen LP. Colonization and infection by Helicobacter pylori in humans. Helicobacter. 2007;12:12-15. doi:10.1111/j.15235378.2007.00574.x

113. Narayanan M, Reddy KM, Marsicano E. Peptic ulcer disease and Helicobacter pylori infection. Mo Med. 2018;115(3):219.

114. Paniker CJ. Paniker's Textbook of Medical Parasitology. JP Medical Ltd; 2017.

115. Windle HJ, Kelleher D, Crabtree JE. Childhood Helicobacter pylori infection and growth impairment in developing countries: a vicious cycle? Pediatrics. 2007;119(3):e754-e759. doi:10.1542/peds.20062196

\section{Publish your work in this journal}

Infection and Drug Resistance is an international, peer-reviewed openaccess journal that focuses on the optimal treatment of infection (bacterial, fungal and viral) and the development and institution of preventive strategies to minimize the development and spread of resistance. The journal is specifically concerned with the epidemiology of antibiotic resistance and the mechanisms of resistance development and diffusion in both hospitals and the community. The manuscript management system is completely online and includes a very quick and fair peerreview system, which is all easy to use. Visit http://www.dovepress.com/ testimonials.php to read real quotes from published authors. 